\title{
The Role of the Epiglottis in the Swallow Process after a Partial or Total Glossectomy Due to a Neoplasm
}

\author{
Ludmiła Halczy-Kowalik • Mieczysław Sulikowski • \\ Rościsław Wysocki • Violetta Posio • \\ Robert Kowalczyk • Anna Rzewuska
}

Received: 7 November 2009/Accepted: 22 January 2011/Published online: 29 March 2011

(C) The Author(s) 2011. This article is published with open access at Springerlink.com

\begin{abstract}
Repeatable epiglottic movement patterns were recorded during a videofluoroscopic swallow evaluation of 95 patients who had undergone a total or partial glossectomy due to a neoplasm. Because no epiglottic function assessment was performed preoperatively, for the purpose of this study it was assumed that epiglottic mobility was "normal" during this time and that all abnormalities found afterward resulted from the growth of the neoplasm and the glossectomy. It was noted that in the early postoperative period, absence of epiglottic movement was accompanied by aspiration and made swallowing incompetent in a majority of cases (9 of 10). A correlation of movement between the epiglottis and the extent of oral tissue excision was found. Epiglottic mobility was evaluated as "normal" in $72 \%$ of the patients, i.e., in 67 of $91(74 \%)$ patients after a partial or nearly total glossectomy and in 1 of 4 people who underwent a total glossectomy. In the subgroup (16\%) of patients who underwent a total or nearly total glossectomy and then had videofluoroscopic examinations, $60 \%$ of the cases had normal epiglottic movements and $40 \%$ had an immobile epiglottis. Compensatory mechanisms implemented by the patients on their own initiative, such as additional swallows and prolonged apnea during
\end{abstract}

L. Halczy-Kowalik ( $\square)$

Independent Laboratory of Postoperative Rehabilitation in

Maxillofacial Surgery, Pomeranian Medical University, 71-114

Szczecin, Al. Powstańców Wlkp. 72, Szczecin, Poland

e-mail: sprpchst@sci.pam.szczecin.pl

M. Sulikowski - R. Kowalczyk · A. Rzewuska

Clinic of Maxillofacial Surgery, Pomeranian Medical University,

Szczecin, Poland

R. Wysocki · V. Posio

Department of Imaging Diagnostics and Interventional

Radiology, Pomeranian Medical University, Szczecin, Poland deglutition, enabled them to avoid aspiration. However, upward head movement and downward chin tilting during deglutition as compensatory mechanisms used by patients with no epiglottic movement did not reduce the aspiration risk in the early postoperative period and were found to accompany incompetent swallowing attempts.

Keywords Epiglottic movement - Swallowing competence $\cdot$ Compensatory mechanisms $\cdot$ Oral cavity cancer · Glossectomy · Deglutition · Deglutition disorders

Change of the upright vertical resting position of the epiglottis into a horizontal one accompanied by the downfolding of its free border constitutes a part of the mechanism protecting the lower airways during a normal breathing procedure. The mechanism that starts with the initiation of the pharyngeal stage of swallowing consists of a forward-upward movement of the hyoid bone, backward movement of the tongue, closure of the larynx by the epiglottis, and closure of the vocal cords. Exclusion of one or more components of this protective mechanism after a partial or total glossectomy due to a neoplasm may allow food leakage or aspiration into the lower airways. In order to avoid aspiration, patients change the position of their heads with respect to their bodies, hold their breath long before swallowing and use multiple swallows to clear the pharyngeal residue. The efficiency of the mechanisms used by patients to cope with deglutition disorders is varied and sometimes difficult to predict.

Unsuccessful re-education of patients in how to swallow after a partial or total glossectomy due to a neoplasm made us review retrospectively all of the videofluoroscopic records of swallowing in rehabilitated patients. We took particular account of the role of the epiglottis in the new 
deglutition model being created. The anatomy of the epiglottis, its age-related changes, the topography of the surrounding tissues, and the factors affecting its movement patterns were evaluated and described in a detailed way [1-4]. Irregularities in epiglottic movement accompanying deglutition disorders have already been observed and analyzed by various authors. In a couple of studies they were described as one of the most significant factors to increase the risk of aspiration [5, 6]. However, there have been a few reports on the influence of the epiglottic movement pattern on the efficiency of patients' re-education in swallowing after a partial or total glossectomy due to a neoplasm.

\section{Patients and Methods}

\section{Patients}

The subjects were 95 patients, including 28 women aged 20-78 years $($ mean $=46.3)$ and 67 men aged 9-79 years (mean $=53.9)$. All had undergone surgical treatment for an oral cavity neoplasm at the Clinic of Maxillofacial Surgery at the Pomeranian Medical University which consisted of a partial or total glossectomy. None of our patients had ever had a condition that affected swallowing efficiency diagnosed or treated before. Hemiglossectomy was performed in 44 patients (46\%), a nearly total glossectomy in $12(12.6 \%)$, and a total glossectomy in 6 $(6.3 \%)$. In all those cases, the quality of the videofluoroscopic records of the act of swallowing allowed for both the evaluation of the epiglottic movement pattern and determination of the time sequence of all actions involved in it. Neither the presurgical medical interview nor the clinical assessment revealed a condition, other than the oral cavity neoplasm, that might involve swallowing disorders.
No videofluoroscopic study had been done before the surgery. The extent of both the oral tissue excision and the reconstruction performed as a one-step surgery are presented in Table 1. Tongue reconstruction was carried out in eight patients, and three patients had restoration of the tongue and floor of the mouth. Frontal, pectoral, and microvascular free flaps from the forearm were used for reconstruction purposes. The mandibular arch was maintained in 13 cases by means of a mandibular locking plate which stabilized in a nearly anatomical position the parts of the bone remaining after a partial mandibulectomy and, at the same time, served as a scaffold for suprahyoid muscles or parts thereof. All the other reconstructions were performed by means of tissues near the postoperative bone defect.

\section{Methods}

Videofluoroscopic evaluation of swallowing was carried out between 2 and 4 weeks postoperatively in patients who had complaints of difficulty swallowing saliva, in whom the extent of tissue excision implied a severe swallowing dysfunction, and in those who were afraid of the removal of the feeding tube and reinitiation of normal feeding.

The images obtained by means of the EDR 700 model (X-ray unit, Medicor Co., Medical Röntgen RT, Budapest, Hungary) were video-recorded. The recordings were made on magnetic video tape using VHS at the rate of 24 frames per second. This enabled us to watch multiple replays of the recording, including slow motion and frame-by-frame. The digital real-time recordings were saved and stored as JPEG files on a computer hard disc, which made it possible for us to evaluate the time sequences of the specific components of the recording. The images taken of the oral and pharyngeal phases of swallowing were both lateral and anterior-posterior views. The patients were seated
Table 1 Extent of oral tissue excision followed by the reconstruction of significant structures

\begin{tabular}{lccc}
\hline Extent of excision & $\begin{array}{l}\text { Total No. } \\
\text { of cases }\end{array}$ & $\begin{array}{l}\text { No. of cases } \\
\text { followed by } \\
\text { reconstruction } \\
\text { or not }\end{array}$ \\
\cline { 2 - 4 } & Yes & No \\
\hline I-Anterior parts of the tongue, mandibular body, and floor of the mouth & 8 & 7 & 1 \\
II-Lateral parts of the tongue, mandibular body, and floor of the mouth & 13 & 6 & 7 \\
III-Hemimandibulectomy and hemiglossectomy & 19 & 8 & 11 \\
IV-Nearly the whole tongue, more than half of the mandible, & 12 & 9 & 3 \\
and anterior and lateral parts of the floor of the mouth & 6 & 5 & 1 \\
V-Total glossectomy & 25 & 2 & 23 \\
VI-Hemiglossectomy & 2 & 5 & 7 \\
VII-Ramus of the mandible, lateral wall of pharynx, & & 52 & 53 \\
and root of the tongue & 95 & 42 \\
Total & & 5
\end{tabular}


comfortably with the head positioned to facilitate to feeding. The study started with the lateral view of the oral cavity (when possible), nasopharynx, midpharynx, laryngopharynx and the cervical part of the esophagus. When it was impossible to have all the above-mentioned structures visible in one image, the oral and pharyngeal phases of swallowing were studied on two different images. One image showed the whole oral cavity, nasopharynx, midpharynx, and part of the laryngopharynx. The other image was supposed to show the posterior part of the oral cavity, midpharynx, laryngopharynx and the cervical part of the esophagus.

For the radiological studies we used a barium suspension. For the first swallowing study, a thin suspension in an amount not exceeding $5 \mathrm{ml}$ was used. If the patient undergoing assessment suffered from a massive aspiration, the study was immediately terminated. If there was no risk of serious aspiration, the patients underwent further swallowing evaluations carried out by means of an increased amount of suspension ranging from 20 to $25 \mathrm{ml}$. During the evaluations, the consistency of the suspension was pudding- or paste-like and the patients were free to decide how many times they wanted to swallow.

The swallowing act was analyzed based on findings included in two reports by Dodds et al. [7, 8] in which the methods of assessment of both normal and abnormal oral and pharyngeal phases of swallowing were described. The following criteria were taken into account: labial closure, palatopharyngeal closure, oral transit of the food bolus, mobility of the tongue, postswallow oral residue, additional swallows used for emptying the oral cavity, oral trajectory of the bolus, body posture and head movement during the swallows, preswallow leakage, mobility of the epiglottis, glossopharyngeal closure, pharyngeal trajectory of the bolus, additional swallows used for emptying the laryngopharynx, postswallow laryngopharyngeal residue, postswallow leakage into the lower airways, protection of the lower airways, intraswallow leakage, and aspiration.

The records were analyzed instantly on a real-time basis by a team comprising the patient, two radiologists, and a clinician (maxillofacial surgeon). The radiologists stratified the swallowing competence of patients using such descriptions as:

1. incompetent (massive and persistent aspiration or lack of dynamic propulsion of the food bolus from lips to esophagus making the oral feeding impossible);

2. poorly functional (no aspiration in the course of the study; impairment of actions involved in the oral and pharyngeal phases of swallowing that may result in aspiration owing to the penetration of the laryngeal vestibule and postswallow vallecular and pyriform sinus residue; need to initiate the re-education in swallowing before the feeding-tube removal);
3. moderately functional (low risk of aspiration; impaired actions involved in the oral and pharyngeal phases of swallowing performed owing to the compensatory mechanisms spontaneously applied by the patient or suggested during the study; possibility of oral feeding provided that the feeding period lasts long enough and learned compensatory mechanisms are temporarily applied);

4. functional (no aspiration risk; "safe" transit of the bolus from the oral cavity into the pharynx; sufficient autoregulatory mechanisms used by the patient; possibility of starting oral feeding without any limitations);

5. correct (insignificant impairment of the activities involved in swallowing with no effect upon the course of this act; no change in the dynamics; no need to implement autoregulatory mechanisms).

The above-mentioned evaluation phase was aimed at finding the answer to the following question: "Can we initiate oral feeding of the patient or shall we maintain tube-feeding while the patient's re-education of swallowing is carried through?"

The video records were analyzed frame-by-frame without the participation of the patient. A qualitative evaluation of the swallowing act was carried out, including such parameters as mobility of the tongue and epiglottis; labial, palatopharyngeal, and glossopharyngeal closures, and postswallow pharyngeal residue. A value from 1 to 4 was assigned to each aspect, where 1 was "normal" and 4 was "absent." The criteria of evaluation also included patients' postures during the swallows, additional swallows affected by them while holding their breath, and the bolus trajectory. The said criteria were assigned values from 1 to 4 as well. Quantitative features of the act of swallowing, such as the moment of initiation, termination, and the duration of the formation of the bolus, oral and pharyngeal transfer, laryngeal closure, and opening of the upper esophageal sphincter, were described as moments in time or time spans. The point of reference for time measurements was referred to as $0.00 \mathrm{~s}$ and corresponded to initiation of the pharyngeal phase.

The evaluation phase mentioned above was aimed at identifying circumstances in which the penetration and/or aspiration occurred and for formulating indications for the re-education of swallowing.

The maxillofacial surgeon used her own 100-point scale [9] during the course of the observations of both the patient and the videofluoroscopic image of the swallowing act while keeping in mind the postoperative tissue damage, the compensatory mechanisms used by the patient, and the symptoms of impaired swallowing such as coughing, choking, and drooling. For the purpose of this evaluation, 
ten composed actions performed by the oral cavity, throat, and esophagus structures were taken into account. The activities on which swallowing efficiency depends include:

1. tight lip closure (enhanced by an increased function of the facial expression muscles, pressing the mandible against the maxilla, putting the remaining part of the tongue between the lips, sucking the fluid in, and pressing the lower lip against the upper one with the hand);

2. ability to move the food bolus toward the dorsum of the tongue (enhanced by a sucking-like movement of the cheeks);

3. ability to move the food bolus along the dorsum of the tongue toward the pharynx (enhanced by several upward movements of the head, repeated pressing of the mandible against the maxilla, multiple swallows);

4. tight glossopalatal closure (enhanced by pressing the mandible against the maxilla, sucking in the cheeks);

5. tight nasopharyngeal closure (enhanced by tilting the head at the moment of swallowing in the direction opposite the operated tissues);

6. ability of the tongue to propel food bolus into the pharynx (enhanced by sucking in the cheeks, fast upward movement of the head, and multiple swallows accompanying one apnea episode);

7. efficient airway closure (enhanced by swallowing with down tilted chin or the head rotated in the direction opposite the operated part of the tissues, holding the breath during the swallow preparation and grunting after the swallow, an additional swallow during a single apnea episode);

8. competent transport of the bolus through the pharynx (enhanced by multiple swallows accompanying a single apnea episode and made by means of facial expression muscles and suprahyoid muscles as well as by the head movements);

9. opening of the upper esophageal sphincter at an adequate time and of an adequate duration (enhanced by forward movement of the head, pressing the mandible against the maxilla, and increased contraction of the expression muscles and suprahyoid muscles);

10. unobstructed breath immediately following the swallow (enhanced by a deep breath preceding the swallow, multiple swallows during a single apnea episode, strong exhalation, and an additional swallow).

The maximum number of points to be scored for each of those activities was 10 . When an activity was either not completed or was completed by means of supplementary mechanisms, 5 points were scored, and when a specific activity was absent, 0 points were given. All of the points scored were then summed up. The total score was the outcome of both a clinical and a radiological evaluation of the swallowing efficiency determined at the end of each examination.

A joint analysis of the descriptive evaluation results and the number of points scored revealed a repeatable regularity, and thus the established radiological description "incompetent" corresponded with a score of 0-20 points, "poorly functional" with 25-45 points, "moderately functional" with 50-65 points, "functional" with 70-85 points, and "correct" with 90-100 points.

Discontinuous variables were verified by means of a $\chi^{2}$ test, continuous variables by means of the Mann-Whitney test, and correlations among variables by means of the Spearman's rank correlation coefficient. The results were described using $R$ correlation coefficients and $P$ probability. In all of the tests, $P<0.05$ was considered to be statistically significant while $0.05<P<0.10$ was the limit of statistical significance.

\section{Results}

The course of the act of swallowing after a partial or total glossectomy due to a neoplasm was evaluated while taking into account such parameters as lip closure, tongue mobility, bolus formation, oral transfer, palatopharyngeal closure, glossopharyngeal closure, oral residue, pharyngeal residue, additional swallows, body posture, head movements during deglutition, airway closure, pre-, intra-, and postswallow leakage, and aspiration. The evaluation performed referred to the epiglottic movement patterns. Table 2 gives the statistically significant correlations between epiglottic movement patterns and phases of deglutition. At the oral phase of deglutition, the strongest relationship was found to exist between tongue mobility and epiglottic movement patterns (a correlation coefficient of $0.42, P=0.00003$ ).

The initiation of the pharyngeal phase was not delayed in only three cases, and in all three patients the epiglottic movement proved to be correct. A delayed pharyngeal phase initiation (PSI) was observed in 92 of 95 patients who had undergone a partial or total glossectomy. The delayed PSI was, to a highly statistically significant extent, correlated to limited epiglottic movement (a correlation coefficient of $0.42, P=0.00043)$. A normal epiglottic movement was recorded in $90 \%$ of the patients in whom the PSI occurred before the lapse of the first $0.1 \mathrm{~s}$ of its duration, and in 50\% of the patients in whom the PSI was recorded after the lapse of $0.6 \mathrm{~s}$. Absence of epiglottic movements was noted in the cases where the delay in the PSI was the most considerable. 
Table 2 Statistically significant correlations between epiglottic movement patterns and the swallow process

\begin{tabular}{|c|c|c|c|c|c|c|c|}
\hline \multirow[t]{2}{*}{ Swallowing stages } & \multirow[t]{2}{*}{ Performance } & \multicolumn{4}{|c|}{ Epiglottic movement pattern } & \multicolumn{2}{|c|}{$\begin{array}{l}\text { Spearman's rank } \\
\text { correlation coefficient }(P)\end{array}$} \\
\hline & & $\begin{array}{l}\text { Normal } \\
(n=68)\end{array}$ & $\begin{array}{l}\text { Horizontal } \\
(n=9)\end{array}$ & $\begin{array}{l}\text { Posterior } \\
(n=5)\end{array}$ & $\begin{array}{l}\text { Absent } \\
(n=10)\end{array}$ & & \\
\hline \multirow[t]{2}{*}{ Labial closure } & Full & $60(88.24 \%)$ & $8(88.89 \%)$ & $3(60.0 \%)$ & $6(60.0 \%)$ & 0.23 & 0.02593 \\
\hline & Impaired & $8(11.76 \%)$ & $1(11 \%)$ & $2(40.00 \%)$ & $4(40.00 \%)$ & & \\
\hline \multirow[t]{3}{*}{ Tongue mobility } & Normal & $17(25.00 \%)$ & $3(33.33 \%)$ & $0(0.00 \%)$ & $0(0.00 \%)$ & 0.42 & 0.00003 \\
\hline & Impaired & $42(61.76 \%)$ & $4(44.44 \%)$ & $1(20.00 \%)$ & $2(20.00 \%)$ & & \\
\hline & Absent & $9(13.244 \%)$ & $2(22.22 \%)$ & $4(80.00 \%)$ & $8(80.00 \%)$ & & \\
\hline \multirow[t]{2}{*}{ Glossopharyngeal closure } & Full & $47(70.15 \%)$ & $5(55.56 \%)$ & $0(0.00 \%)$ & $1(10.00 \%)$ & 0.40 & 0.00009 \\
\hline & Impaired & $20(29.85 \%)$ & $4(44.44 \%)$ & $5(100.00 \%)$ & $9(90.00 \%)$ & & \\
\hline \multirow[t]{2}{*}{ Airway closure } & Complete & $68(100 \%)$ & $7(77.78 \%)$ & $0(0.00 \%)$ & $1(10 \%)$ & 0.83 & 0.00000 \\
\hline & Incomplete & $0(0.00 \%)$ & $2(22.22 \%)$ & $5(100.00 \%)$ & $9(90.00 \%)$ & & \\
\hline Preswallow leakage & & $0(0.00 \%)$ & $0(0.00 \%)$ & $0(0.00 \%)$ & $4(40.00 \%)$ & 0.27 & 0.0001 \\
\hline Intraswallow leakage & & $5(55.56 \%)$ & $1(33.33 \%)$ & $1(33.33 \%)$ & $2(20.00 \%)$ & 0.17 & 0.17053 \\
\hline Postswallow leakage & & $4(44.44 \%)$ & $2(66.67 \%)$ & $2(66.67 \%)$ & $4(40.00 \%)$ & 0.37 & 0.0003 \\
\hline Aspiration & & $0(0.00 \%)$ & $0(0.00 \%)$ & $1(10.00 \%)$ & $9(90.00 \%)$ & 0.95 & 0.00001 \\
\hline \multirow{4}{*}{$\begin{array}{l}\text { Postswallow pharyngeal } \\
\text { residue }\end{array}$} & Absent & $55(82.09 \%)$ & $5(55.56 \%)$ & $1(20.00 \%)$ & $3(4.69 \%$ & & \\
\hline & VR & $6(8.96 \%)$ & $0(0.00 \%)$ & $0(0.00 \%)$ & $3(30.00 \%)$ & 0.20 & 0.09343 \\
\hline & VR and PSR & $5(7.46 \%)$ & $2(22.22 \%)$ & $3(60.00 \%)$ & $2(20.00 \%)$ & 0.40 & 0.00029 \\
\hline & DHR & $1(1.49 \%)$ & $2(22.22 \%)$ & $1(20.00 \%)$ & $2(20.00 \%)$ & 0.49 & 0.00001 \\
\hline \multirow{3}{*}{$\begin{array}{l}\text { Head movement during } \\
\text { swallowing }\end{array}$} & Hardly detectable & $55(8.88 \%)$ & $6(66.67 \%)$ & $3(60.00 \%)$ & $5(50.00 \%)$ & & \\
\hline & Upward & $9(13.24 \%)$ & $2(22.22 \%)$ & $1(20.00 \%)$ & $4(40.00 \%)$ & 0.23 & 0.03648 \\
\hline & Downward & $4(5.88 \%)$ & $1(11.11 \%)$ & $1(20.00 \%)$ & $1(10.00 \%)$ & 0.23 & 0.03648 \\
\hline \multirow[t]{2}{*}{ Additional swallows } & Absent & $57(85.07 \%)$ & $54(55.56 \%)$ & $1(20.00 \%)$ & $3(30.00 \%)$ & & \\
\hline & One or more & $10(14.93 \%)$ & $4(44.44 \%)$ & $4(80.00 \%)$ & $7(70.00 \%)$ & 0.48 & 0.00000 \\
\hline
\end{tabular}

$V R$ vallecular residue, $P S R$ pyriform sinus residue, $D H R$ diffuse hypopharyngeal residue

Epiglottic Movement Patterns $(N=95)$

Normal epiglottic movement (horizontal position of the epiglottis with a complete downfolding of its free border) was observed in 68 of 95 cases (72\%), horizontal tilt in 9 cases, hardly detectable posterior translocation in 5 cases, and absence of movement in 10 cases. Movements difficult to assess were registered in three patients in whom the margin required for oncological safety reached pre-epiglottic valleculae. The three patients were not taken into account during evaluation of the results. No statistically significant relationship was found to exist between the sex or age of the patients, time since surgery, or type of neck surgery involving the lymphatic system and epiglottic movement patterns. The extent of excision of the oral cavity structures (Fig. 1) exerted a statistically significant influence on epiglottic movement $(P=0.00637)$.

Normal Epiglottic Movement $(N=68)$

In 68 patients (72\%) who had undergone surgery for oral cavity cancer, irrespective of the extent of tissue excision a normal epiglottic movement was observed. In most cases, normal epiglottic movement occurred following the excision of the lateral part of the tongue, floor of the mouth, and adjacent parts of the mandibular body. Actions involved in the oral phase of swallowing such as tight lip closure, bolus formation, oral transfer, or glossopalatal closure were impaired but still the airway closure was tight.

Penetration occurred in $13 \%$ of patients. No aspiration cases were found. Postswallow residue was noted in $19 \%$ of subjects, mostly in the pre-epiglottic valleculae and pyriform sinuses. Nineteen percent of the patients changed the position of their head with respect to their body while swallowing and $15 \%$ used additional swallows (Table 2). Despite a delay in the initiation of the pharyngeal phase, the swallowing was "functional" (Table 3).

Abnormal Epiglottic Movement $(N=24)$

Abnormal epiglottic movement was observed in 24 (26\%) patients. In 9 of them the epiglottis tilted horizontally, in 5 a hardly detectable posterior translocation was recorded, and in 10 cases it did not move at all. The horizontal tilt 
Fig. 1 Epiglottic movement pattern

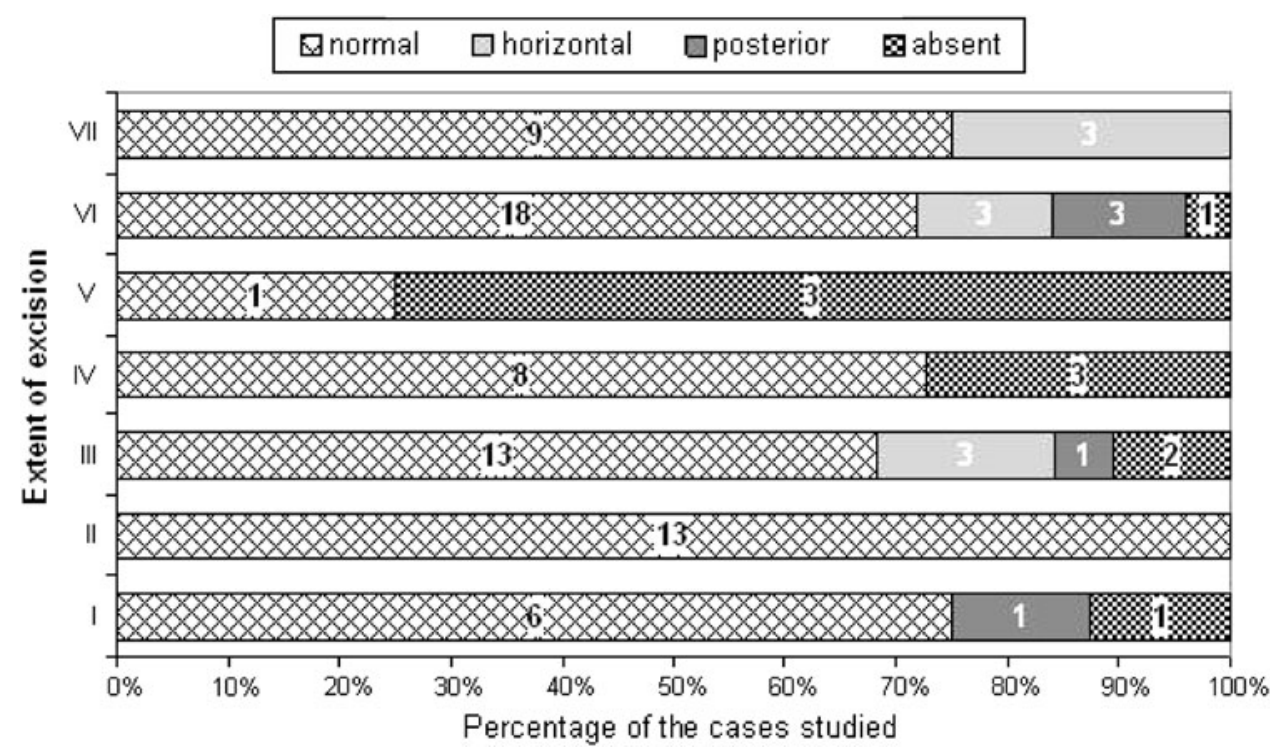

Table 3 Statistically significant correlations between epiglottic movement patterns and both the temporal sequence of components of the swallow process and swallowing competence

\begin{tabular}{|c|c|c|c|c|}
\hline \multicolumn{4}{|c|}{ Epiglottic movement pattern } & \multirow{2}{*}{$\begin{array}{l}\text { Spearman's rank correlation } \\
\text { coefficient }\end{array}$} \\
\hline $\begin{array}{l}\text { Normal } \\
(n=68)\end{array}$ & $\begin{array}{l}\text { Horizontal } \\
(n=9)\end{array}$ & $\begin{array}{l}\text { Posterior } \\
(n=5)\end{array}$ & $\begin{array}{l}\text { Absent } \\
(n=10)\end{array}$ & \\
\hline Average $\pm \mathrm{SD}$ & Average $\pm \mathrm{SD}$ & Average \pm SD & Average $\pm \mathrm{SD}$ & $R$ \\
\hline
\end{tabular}

Temporal sequence of actions involved in the pharyngeal stage of swallowing

$\begin{array}{lcccccc}\text { Initiation of the pharyngeal stage } & 0.40 \pm 0.63 & 0.70 \pm 0.89 & 0.98 \pm 1.00 & 1.07 \pm 0.74 & 0.36 & 0.00043 \\ \text { Laryngeal closure } & 0.44 \pm 0.56 & 0.71 \pm 1.16 & 0.99 \pm 1.03 & 0.69 \pm 0.72 & 0.30 & 0.00536 \\ \text { Opening of the upper esophageal sphincter } & 0.62 \pm 0.57 & 1.12 \pm 1.02 & 1.31 \pm 0.85 & 2.04 \pm 1.16 & 0.47 & 0.00001 \\ \text { Closure of the upper esophageal sphincter } & 1.00 \pm 0.62 & 1.43 \pm 1.04 & 1.50 \pm 0.97 & 2.61 \pm 1.32 & 0.39 & 0.00027 \\ \text { Opening of the larynx } & 1.54 \pm 1.53 & 2.01 \pm 1.21 & 3.18 \pm 2.33 & 3.71 \pm 2.70 & 0.41 & 0.00010 \\ \text { Duration of actions involved in the pharyngeal stage of swallowing } & & & \\ \text { Time for pharynx emptying } & 0.54 \pm 0.37 & 0.53 \pm 0.43 & 0.83 \pm 0.55 & 1.88 \pm 1.36 & 0.27 \\ \text { Time for holding breath } & 1.16 \pm 1.26 & 1.69 \pm 1.72 & 2.27 \pm 2.95 & 3.07 \pm 2.83 & 0.19 \\ \text { Pharyngeal stage duration } & 1.52 \pm 1.35 & 2.42 \pm 1.69 & 3.73 \pm 2.61 & 3.56 \pm 2.57 & 0.42 & 0.00926 \\ \text { Swallowing competence } & 75.00 \pm 15.81 & 65.00 \pm 18.73 & 38.00 \pm 2.55 & 32.50 \pm 17.4 & -0.50 & P=0.0000 \\ & & & & \end{array}$

was found mostly in subjects who underwent hemiglossectomy and hemimandibulectomy. A total glossectomy was performed on 6 patients and a nearly total glossectomy was performed on 12 patients, although we failed to evaluate 3 of them. In 9 of the remaining 15 patients, the epiglottic movement was found to be normal and in 6 no movement was observed. Actions involved in the oral phase of swallowing such as tight lip closure, bolus formation, oral transfer, and glossopalatal closure were considerably impaired or absent. Airway closure was inefficient. Penetration was recorded in $67 \%$ of people with abnormal epiglottic movements and aspiration was recorded in $42 \%$. Aspiration was found mainly in those whose epiglottis remained immobile in the course of swallowing.
The presence of postswallow residue in the pre-epiglottic valleculae, pyriform sinuses, and pharynx was noted in $62.5 \%$ of subjects. In $42 \%$ of cases patients changed the position of their head with respect to their body while swallowing, and in $63 \%$ they used additional swallows (Table 2). A delay in the initiation of the pharyngeal phase of deglutition was 2.5 times longer and swallowing efficiency was almost 2.5 times worse than those in patients enjoying normal epiglottic movements (Table 3 ).

A joint analysis of epiglottic movement patterns and the moments of initiation and termination as well as the duration of the acts constituting the oral phase of swallowing enabled us to notice the following correlation: the more abnormal the epiglottic movement pattern was, the 
more delayed the laryngeal closure and the pharyngeal sphincter opening were, the longer the time needed for emptying the throat was, and the longer the pharyngeal phase and swallowing apnea (although statistically insignificant) were (Table 3 ).

The correlation between the swallowing competence evaluation carried out by means of a 100-point scale and the epiglottic movement pattern $(R=-0.50, P=0.0000)$ was highly statistically significant. An average rating of swallowing competence for patients with normal epiglottic movements was 2.5 times higher than its value for subjects with an absence of epiglottic movements (Table 3).

Following a partial or total glossectomy patients were tubefed in the early postoperative period. At the time preceding the videofluoroscopic evaluation of swallowing, most had already started to apply their own methods of saliva swallowing without the risk of aspiration. Some of them had even made attempts to drink liquids disregarding the feeding tube. Validity of the compensatory mechanisms used by patients was assessed during the videofluoroscopic studies of the swallowing act. As a result, in several cases patients were advised against using ineffective compensatory mechanisms.

Swallowing with the head tilted backward was noted in 16 patients in whom the oral transfer proved to be incompetent. The patients used this maneuver by their own initiative. It enabled them to transfer the bolus from the oral cavity to the throat and to swallow it safely. In four patients with no epiglottic movements, a preswallow leakage was noted, which then turned into aspiration. In all those cases tube-feeding was temporarily maintained (Fig. 2).

Swallowing with the head tilted downward was noted in 7 patients in whom there was no glossopharyngeal closure.

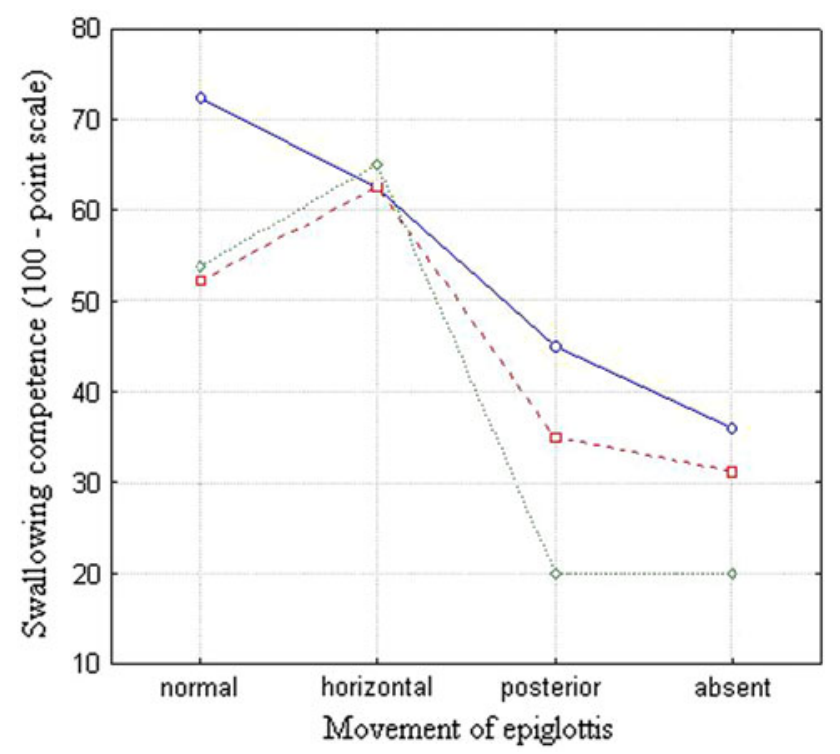

Fig. 2 Head position during swallow: normal (open circle), up (open square), down (open diamond)
In five cases this maneuver enabled the patient to swallow safely, but in two cases in which the epiglottic movements were absent, intraswallow leakages occurred, which then turned into aspiration. In those cases the tube-feeding also was maintained (Fig. 2).

In all of the examined subjects, swallowing one dose of liquid was accompanied by an apnea lasting 0.15-8.08 s. A prolonged apnea $(>0.6 \mathrm{~s})$ was noted in 74 patients. An apnea lasting 0.61-1.19 $\mathrm{s}$ was reported in the cases in which the highest swallowing competence (irrespective of the epiglottic movement pattern) was observed. The said apnea duration was more important for the swallowing competence in patients with absent or hardly detectable epiglottic movements. In subjects with absent epiglottic movements, a higher swallowing competence was found to be accompanied by an apnea lasting more than $1.20 \mathrm{~s} \mathrm{(Fig.} \mathrm{3).}$

Additional swallows were used by 25 patients who attempted to clear the pharynx during one apnea episode. The more profound the epiglottic movement impairment was, the more the subjects used additional swallows. In the case of an absence of epiglottic movements, additional swallows did not always ensure pharynx emptying before the opening of the larynx. In such circumstances, the compensatory mechanism did not protect the patients against aspiration and accompanied incompetent swallowing (Fig. 4).

\section{Discussion}

A partial or total resection of the tongue and neighboring tissues due to a neoplasm may make regular oral feeding impossible or more difficult. Since the $1960 \mathrm{~s}$,

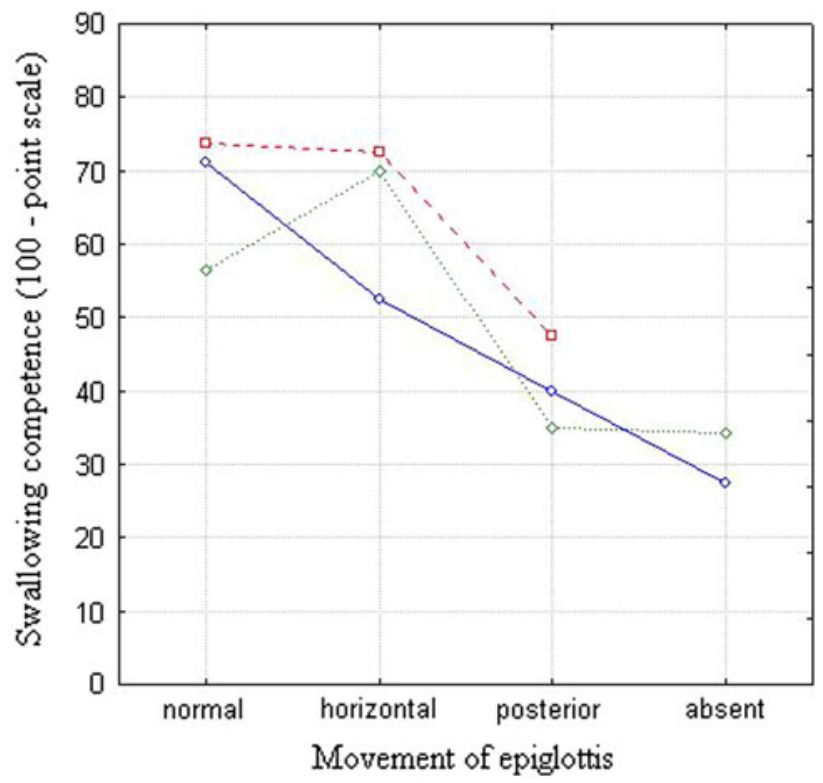

Fig. 3 Time of larynx closure: $\leq 0.60$ (open circle), 0.61-1.19 (open square), $\geq$ (open diamond) 


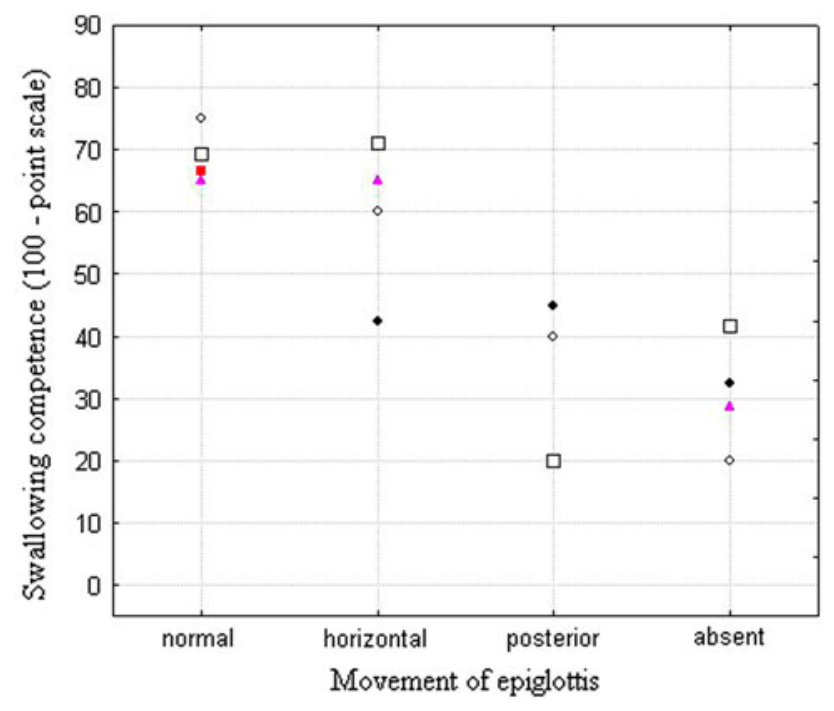

Fig. 4 Additional swallows: 0 (open circle), 1 (open square), 2 (open diamond), 3 (open triangle), $\geq 4$ (filled diamond)

reconstructions of excised structures by means of myocutaneous distal and free flaps using microsurgical techniques have been described. Clinical and, since the 1980 s, videofluoroscopic evaluations of the oral cavity and throat functions were usually carried out several months after surgery that consisted of tumor removal and respective reconstruction. The results of such evaluations show that the said functions are only dependent, to a certain extent, on the selected method of reconstruction, but to a larger extent they are dependent on the scope of tissue excision and the degree to which its mass was reconstructed [10, 11]. Reconstruction of the tongue mass after a hemiglossectomy, where the tongue is resected lengthwise, may be beneficial for the act of swallowing and detrimental for speech generation because of the limited mobility of the remaining part of the tongue [12]. Due to the reinnervation of the free flap, which is used for the tongue restoration, the functional reconstruction of this structure constitutes an evidence-based method leading to an improvement of impaired functions of the oral cavity after a several-monthlong rehabilitation [13-15]. All researchers dealing with this issue used to underline the significance of the period after surgery as well as the need of an early, long-lasting, and multidisciplinary re-education in swallowing [16-19]. Reconstructions performed by us included both restoring the whole tongue or more than half of the tongue and floor of the mouth as well as maintaining the mandibular continuity.

In the early postoperative period, all patients operated on by us found it difficult to swallow saliva. A videofluoroscopic evaluation was the first one that was used to decide on the restoration of oral feeding. In the period preceding the evaluation, patients were not subjected to an intensive reeducation in swallowing, however, the majority of them made attempts to swallow saliva in order to avoid the risk of aspiration. Some of the patients tried to drink liquids by their own initiative and most of them managed to do it. Nevertheless, others were unable to drink a 5-ml dose of contrast medium during a videofluoroscopic evaluation performed 2-4 weeks after the surgery. Penetration was noted in 25 subjects, aspiration in 10 , and postswallow residue in 27. Aspiration occurred in $42 \%$ of the cases of impaired epiglottic movement patterns (including a posterior movement or the absence of movement). There was no case of aspiration in patients with a normal movement pattern. Intra- or postswallow penetration was noted in $25 \%$ of patients with an abnormal epiglottic movement and in $13 \%$ with a normal one. Preswallow penetration was recorded in four patients in whom the epiglottic movement was absent, and in all those cases the penetration turned into aspiration. A strong correlation $(R=0.95, P=$ $0.00001)$ implied that the absence of a normal epiglottic movement pattern was a factor of considerable importance for aspiration occurrence.

Perlman [5] and Garon [6] presented findings of results of a videofluoroscopic evaluation of swallowing in patients with a number of admitted diagnostic categories. They reported a higher incidence(in more than 55\% of subjects) of aspiration accompanying impaired epiglottic movements than was found in the group participating in our research. They also observed the occurrence of aspiration in patients enjoying normal epiglottic movement patterns. These authors noted an important interdependence between both the course of the oral phase of deglutition and the epiglottic movement patterns and between the epiglottic movement patterns and the amount of postswallow residue.

The correlation of disorders of the oral phase of swallowing to both a limited forward movement of the larynx and limited angular deviation of the epiglottis during deglutition in subjects who underwent surgery and radiotherapy due to an oral cavity neoplasm was demonstrated by Kitano [20] in a Cine-MRI performed within 1 month after termination of the treatment.

In our research, which was focused on subjects who underwent a partial or total glossectomy due to a neoplasm, mobility of the tongue (its remaining part or the structure resulting from tongue reconstruction surgery) was the most important parameter of the oral phase of deglutition that affected epiglottic movement $(R=0.42, P=0.00003)$. Because of the reduced mass of the tongue and its limited mobility, impaired glossopharyngeal closure and lack of tight airway closure were noted during swallowing.

Absence of the labial seal was another parameter of the oral phase of swallowing whose relationship with epiglottic movement was statistically significant $(R=0.23, P=$ $0.02593)$. It resulted most frequently from the resection of 
the part of the mandibula affected by neoplasm and was recorded in the early postoperative period irrespective of whether the mandibular arch had been reconstructed or not.

The moments of initiation and termination as well as duration of the acts constituting the oropharyngeal phase of swallowing in controls were determined [21, 22]. Based on a topokinetic analysis of the videofluoroscopic evaluation records, a time-dependent variation of the positions of the anatomical structures taking part in the physiological act of swallowing was described [23]. Kendall et al. [24] and Morton et al. [25] stressed the importance of extending the time of laryngeal closure necessary for pharynx emptying in patients with swallowing dysfunction caused by neurological disorders.

In the patients in our study, the moment of laryngeal closure was delayed with respect to the initiation of the oral phase. The more abnormal the epiglottic movement was, the more significant a delay we noted. The only exception was in a group of subjects with absent epiglottic movements where the delay in laryngeal closure was relatively short. It can be presumed that repeatable aspirations during swallowing of saliva made the patients affected by this disorder learn to close their larynx earlier.

In almost all of our subjects, the breath-holding time was extended, and it was observed that longer extensions accompanied more abnormal epiglottic movement patterns. Although this correlation is hardly statistically significant $(P=0.07534)$, it is still an important guideline at the beginning of re-education in swallowing.

The ability of patients to learn postural and breathing maneuvers that enable them to improve their swallowing competence helps them maintain oral feeding skills whether they have temporary or permanent impairment of swallowing functions of the oropharyngeal cavity. Although the above-mentioned examples of a deliberate generation of new models of swallowing refer to the reeducation of patients from various diagnostic categories on how to swallow, their use is aimed at coping with specific disorders such as profoundly impaired oral transfer, incomplete glossopharyngeal closure, or delayed initiation of the pharyngeal phase of deglutition [26-31].

The postural maneuvers, additional swallows, and extended apnea that we noted in our patients were not learned techniques. They were individual methods used by patients during their attempts to swallow saliva. All of the subjects with abnormal epiglottic movement patterns and less than one fifth of the subjects with normal epiglottic movements used the described methods.

The effectiveness of those autoregulatory maneuvers in improving swallowing competence varied. They used to accompany a nearly or moderately functional swallowing performance in patients with normal or horizontal epiglottic movement patterns as well as poorly functional or incompetent swallowing in patients with a hardly detectable posterior movement or absence of movement of the epiglottis.

Prolonged apnea as implemented by the subjects was advantageous for swallowing competence. An apnea lasting longer than $1.20 \mathrm{~s}$ was noted in patients who enjoyed competent swallowing in spite of an absence of the epiglottic movement.

Additional swallows performed during a single apnea were recorded in $15 \%$ of those with normal epiglottic movement patterns and in $63 \%$ of cases with abnormal ones. In almost all of the subjects using additional swallows, the swallowing performance was at least poorly functional.

Swallowing with the head tilted backward was a maneuver used by $13 \%$ of patients with normal epiglottic movements and by $29 \%$ of patients with abnormal epiglottic movements. In four subjects with absent epiglottic movements, preswallow leakage and aspiration occurred while they attempted to swallow a 5-ml dose of contrast medium during the first evaluation, even though they managed to swallow saliva. The next attempted swallow of a smaller amount of contrast medium, performed as a supraglottic swallow following the instructions of the person carrying out the research, was accompanied by preswallow leakage which was then coughed out. It was assumed that the risk of aspiration in those patients was considerable so feeding through an esophageal tube was maintained and re-education in swallowing was initiated.

The maneuver of swallowing whereby the chin is tilted toward the chest was used by 3 of 24 patients with abnormal epiglottic movement patterns. In two patients with a hardly detectable or absent movement of the epiglottis, intraswallow leakage was recorded which later on turned into aspiration. Another swallow, performed using the supraglottic swallow, of contrast medium in a smaller amount was accompanied by an insignificant postswallow leakage that the patient failed to cough out. Aspiration occurred in both cases, so feeding through an esophageal tube was maintained and re-education of swallowing was initiated.

As a result of analyzing the records of the videofluoroscopic evaluation of deglutition in patients after a partial or total glossectomy due to a neoplasm, the irregularities in the oral phase of deglutition were found to be related to the epiglottic movement patterns. An effect of epiglottic movement patterns on disorders of the pharyngeal phase of deglutition was found. Videofluoroscopic evaluation of swallowing in the early postoperative period is a necessary verification of the autoregulatory mechanisms used by patients during saliva swallowing, before the initiation of normal feeding or before the initiation of re-education in swallowing. This evaluation enables us to define the conditions under which the risk of aspiration becomes lower and to eliminate mechanisms involving a risk of aspiration 
in patients with limited mobility of the epiglottis. While swallowing a larger amount of liquid, both the "head-up" and "head-down" maneuvers allow aspiration in patients with a limited or absent mobility of the epiglottis, even if they manage to successfully swallow saliva. This information is particularly important at the beginning of re-education in swallowing.

Open Access This article is distributed under the terms of the Creative Commons Attribution Noncommercial License which permits any noncommercial use, distribution, and reproduction in any medium, provided the original author(s) and source are credited.

\section{Appendix}

The 100-point scale used for both clinical and radiological assessment of swallowing competence is based on what we know about the extent of tissue excision, healing processes, the smooth swallowing of saliva, simultaneous observation of the patient swallowing the contrast medium, and the radiological image visible on the screen. The ten stages of the swallowing act evaluated by the maxillofacial surgeon in the course of such observation are specified below. The surgeon awarded points according to the following principles: patients able to perform an action scored 10 points, patients unable to perform a certain action comprehensively and who implemented compensatory mechanisms scored 5 points, and patients unable to perform an action in spite of the compensatory mechanism used by them scored 0 . During the evaluation, the following components of the swallowing act were taken into account:

(1) tight lip closure:

- visible complete adherence of the upper lip to the lower one with no anterior leakage of the contrast medium before, during, and after swallowing-10 points,

- visible enhanced function of the expression muscles of the face, pressing the mandible against the maxilla, putting the remaining part of the tongue between the lips, sucking the fluid in, and pressing the lower lip against the upper one with the hand- $\mathbf{5}$ points,

- involuntary leakage of saliva and the contrast medium in spite of the patient's attempts to keep the fluids in the oral cavity- 0 points;

(2) ability to form food bolus on top of the tongue:

- visible accumulation of more than half of the contrast medium volume in the upper part of the oral cavity, above the remaining part of the tongue-10 points,
- visible sucking-like movement of the cheeks, accumulation of less than half of the contrast medium volume in the upper part of the oral cavity -5 points,

- contrast medium pooling at the floor of the mouth, partial leakage into pharynx, accumulation of the remaining part of the contrast medium in the oral cavity- 0 points;

(3) ability to propel the food bolus along the tongue toward the pharynx:

- visible waving movement of the remaining part of the tongue propelling more than half of the contrast medium volume toward the pharynx10 points,

- moving a considerable part of the contrast medium toward the pharynx by means of an upward movement of the head, multiple swallows -5 points,

- contrast medium pooling at the floor of the mouth in spite of an upward movement of the head and multiple swallows, inability to propel the medium into the pharynx $-\mathbf{0}$ points;

(4) tight glossopalatal closure:

- visible complete adherence of the remaining part of the tongue to the palate-10 points,

- visible pressing of the mandible against the maxilla, sucking in the cheeks, approximation of the tongue to the palate, limited area of palatolingual contact $\mathbf{- 5}$ points,

- lack of palatolingual contact in spite of pressing the mandible against the maxilla and sucking in the cheeks-0 points;

(5) tight nasopharyngeal closure:

- visible backward movement and adherence of the velum to the posterior pharyngeal wall-10 points,

- unilateral lack of seal, head tilt at the moment of swallowing in the direction opposite the side lacking nasopharyngeal seal, no leakage into the nasal cavity -5 points,

- lack of velopharyngeal seal, contrast medium entering the nasal cavity- $\mathbf{0}$ points;

(6) ability of the tongue to propel food bolus into the pharynx:

- visible increase in the vertical dimension of the remaining part of the tongue, backward movement of the tongue, transport of the whole bolus into the pharynx-10 points,

- sucking in the cheeks, fast upward movement of the head, multiple swallows accompanying one 
apnea episode, more than half of the contrast medium volume visible in the esophagus- $\mathbf{5}$ points,

- in spite of application of compensatory mechanisms, a considerable part of the food bolus remaining in the laryngopharynx, aspiration of a small amount of contrast medium-0 points;

(7) complete airway closure:

- visible during a so-called "empty swallow" backward movement of the base of the tongue until its contact with the posterior pharyngeal wall and lowered and drawn-back velum, free margin of the epiglottis being drawn downward, the hyoid bone moving forward and upward-10 points,

- swallowing with tilted down chin or head rotated in the direction opposite the operated part of the tissues, holding breath during swallow preparation and grunting after the swallow, expulsion of the contrast medium that penetrated the laryngeal vestibule visible in the screen, no aspiration-5 points,

- swallowing with the head tilted backward, replacing the impaired oral transport with gravitational transport, massive leakage to the laryngeal vestibule resulting in aspiration-0 points;

(8) competent transport of bolus through the pharynx:

- visible transition downward of the food bolus propelled into the pharynx by the tongue, complete nasopharyngeal seal above the bolus, the anterior surface of the epiglottis placed in a horizontal position or tilted with its free margin drawn downward as well as the entry to the esophagus being below the bolus, closed respiratory tract, shape of the bolus gradually changing, with its upper part still outlining the valleculae, middle part being pressed by the pharyngeal muscles, and its lower part entering the esophagus-10 points,

- visible food residue in the oral cavity, extended shape of the food bolus in the pharynx, multiple swallows accompanying one apnea episode and made by means of facial expression muscles and suprahyoid muscles, successful clearance of the oral, valleculae, and pyriform sinus residue-5 points,

- visible food residue in the oral cavity, extended shape of the food bolus in the pharynx, multiple swallows failing to transport the whole food bolus downward into the pharynx, the first inhalation attempt resulting in aspiration of the valleculae and pyriform sinus contrast medium residue-0 points;

(9) opening of the upper esophageal sphincter at an adequate time and for an adequate duration:

- opening of the upper esophageal sphincter before the whole food bolus is visible in the screen filling in the laryngopharynx (the airway being closed), transit of the whole bolus into the esophagus followed by the first postswallow inhalation-10 points,

- visible food residue in the oral cavity, extended shape of the food bolus in the pharynx, bolus head reaching the entrance to the esophagus (the larynx being closed) and getting into the esophagus, multiple swallows of the remaining part of the bolus during a single apnea episode accompanied by forward movement of the head and pressing the mandible against the maxilla as well as contracting facial expression muscles and suprahyoid muscles, complete clearance of the oral cavity and laryngopharyngeal residue $\mathbf{- 5}$ points,

- visible food residue in the oral cavity, extended shape of the food bolus in the pharynx, bolus head being stopped before entry into the closed esophagus and penetrating the laryngeal vestibule, multiple swallows of the remaining part of the bolus during a single apnea episode accompanied by forward and upward movement of the head, both the first delayed opening of the esophagus and its following openings failing to clear the laryngopharyngeal residue, the first postswallow inhalation, preceded by a longlasting apnea episode, being accompanied by aspiration-0 points;

(10) unobstructed breath immediately following the swallow:

- a completely empty oral cavity visible on the screen, the whole food bolus being propelled through the pharynx into the esophagus with the larynx closed, postswallow inhalation-10 points,

- visible food residue in the oral cavity, extended shape of the food bolus in the pharynx, swallow preceded by a deep breath, multiple swallows during a single apnea episode, strong exhalation, grunting and an additional swallow resulting in the first undisturbed breath $\mathbf{- 5}$ points,

- visible food residue in the oral cavity, extended shape of the food bolus in the pharynx, in spite of multiple swallows accompanying a single apnea 
episode, a considerable part of the food bolus being left in the laryngopharynx and then aspired during the first breath-0 points.

All the above descriptions constitute a summary of results of the repeated observations made in 300 cases of patients who suffered from oral cavity cancer and underwent a swallowing evaluation in the period from 1995 to 2010.

\section{References}

1. Yokoyama S, Kano M, Watanabe M, Ogawa H, Omori K. Morphological and histologic examination of the epiglottis: implications for improving epiglottic closure technique. Ann Otol Rhinol Laryngol. 2006;115(1):23-9.

2. Kano M, Shimizu Y, Okayama K, Igami T, Kikuchi M. A morphometric study of age-related changes in adult human epiglottis using quantitative digital analysis of cartilage calicification. Cells Tissues Organs. 2005;180:126-37.

3. Vandaele DJ, Perlman AL, Cassell MD. Intrinsic fibre architecture and attachments of the human epiglottis and their contributions to the mechanism of deglutition. J Anat. 1995;186:1-15.

4. Reidenbach MM. The periepiglottic space: topographic relations and histological organisation. J Anat. 1996;188:173-82.

5. Perlman AL, Booth BM, Grayhack JP. Videofluoroscopic predictors of aspiration in patients with oropharyngeal dysphagia. Dysphagia. 1994;9:90-5.

6. Garon BR, Huang Z, Hommeyer S, Eckman D, Stern GA, Ormiston C. Epiglottic dysfunction: abnormal epiglottic movement patterns. Dysphagia. 2002;17:57-68.

7. Dodds WJ, Stewart ET, Logemann JA. Physiology and radiology of the normal oral and pharyngeal phases of swallowing. AJR Am J Roentgenol. 1990;154:953-63.

8. Dodds WJ, Logemann JA, Stewart ET. Radiologic assessment of abnormal oral and pharyngeal phases of swallowing. AJR Am J Roentgenol. 1990;154:965-74.

9. Halczy-Kowalik L. Disturbances of deglutition taste and stereognosis after the resection of oral cavity neoplasms. Habilitation thesis. Annals of the Pomeranian Medical University, Ann Acad Med Stetinensis Suppl 37, 1997.

10. Krespi YP, Sisson GA. Reconstruction after total or subtotal glossectomy. Am J Surg. 1983;146:486-92.

11. Kimata Y, Sakuraba M, Hishinuma S, Ebihara S, Hayashi R, Asakage T, Nakatsuka T, Harii K. Analysis of the relations between the shape of the reconstructed tongue and postoperative functions after subtotal or total glossectomy. Laryngoscope. 2003;113:905-9.

12. Hsiao HT, Leu YS, Lin C-C. Primary closure versus radial forearm flap reconstruction after hemiglossectomy: functional assessment of swallowing and speech. Ann Plast Surg. 2002;49:612-6.

13. Yamamoto Y. Functional reconstruction of the tongue and deglutition muscles following extensive resection of tongue cancer. Plast Reconstr Surg. 1998;102:993-8.

14. Sanger JR, Campbell ZY, Yousif NJ, Matloub HS. Tongue reconstruction with a combined brachioradialis-radial forearm flap. J Reconstr Microsurg. 2000;16(1):7-13.

15. Urken ML, Buchbinder D, Weinberg H, Viskery C, Sheiner A, Parker R, Schaefer J, Som P, Shapiro A, Lawson W, Biller HF. Functional evaluation following microvascular oromandibular reconstruction of the oral cancer patient: a comparative study of reconstructed and nonreconstructed patients. Laryngoscope. 1991;101:935-50.

16. Schliephake H. Impact of intraoral soft-tissue reconstruction on the development of quality of life after ablative surgery in patients with oral cancer. Plast Reconstr Surg. 2002;109(2) :421-30.

17. McConnel FM, O'Connor A. Dysphagia secondary to head and neck cancer surgery. Acta Otorhinolaryngol Belg. 1994;48:165-70.

18. Furia CL, Carrara-de-Angelis E, Martins NM, Barros AP, Carneiro B, Kowalski P. Video fluoroscopic evaluation after glossectomy. Arch Otolaryngol Head Neck Surg. 2000;126:378-83.

19. Schindler A, Vincon E, Grosso E, Miletto AM, Di Rosa R, Schindler O. Rehabilitative management of oropharyngeal dysphagia in acute care settings: data from a large Italian teaching hospital. Dysphagia. 2008;23:230-6.

20. Kitano H, Asada Y, Hayashi K, Inoue H, Kitajima K. The evaluation of dysphagia following radical surgery for oral and pharyngeal carcinomas by Cine-Magnetic Resonance Imaging (Cine-MRI). Dysphagia. 2002;17:187-91.

21. Kendall KA, McKenzie S, Leonard RJ, Gonçalves MI, Walker A. Timing of events in normal swallowing: a videofluoroscopic study. Dysphagia. 2000;15:74-83.

22. Kim Y, McCullough GH, Asp CW. Temporal measurements of pharyngeal swallowing in normal populations. Dysphagia. 2005;20:290-6.

23. Sulikowski M. The course of pharyngeal phase of the deglutition act under physiological conditions. Computerized topokinetic analysis of roentgenocinematographic images. Central East Eur J Oto-Rhino-Laryngol Head Neck Surg. 1998;2(10):279-85.

24. Kendall A, Leonard RJ, McKenzie S. Airway protection: evaluation with videofluoroscopy. Dysphagia. 2004;19:65-70.

25. Morton R, Minford J, Ellis R, Pinnington L. Aspiration with dysphagia: the interaction between oropharyngeal and respiratory impairments. Dysphagia. 2002;17:192-6.

26. Sulikowski M. Problems in swallowing after partial supraglottic and supracricoid laryngectomy. Otolaryngol Pol. 2001;55(6):605-15.

27. Rasley A, Logemann JA, Kahrilas PJ, Rademaker AW, Paulosky BR, Dodds WJ. Prevention of barium aspiration during videofluoroscopic swallowing studies: value of change in posture. AJR Am J Roentgenol. 1993;160:1005-9.

28. Kahrilas PJ, Logemann JA, Gibbons P. Food intake by maneuver: an extreme compensation for impaired swallowing. Dysphagia. 1992;7:155-9.

29. Welch MV, Logemann JA, Rademaker AW, Kahrilas PJ. Changes in pharyngeal dimensions effected by chin tuck. Arch Phys Med Rehabil. 1993;74:178-83.

30. Logemann JA, Rademaker AW, Paulosky BR, Kahrilas PJ. Effects of postural change on aspiration in head and neck surgical patients. Otolaryngol Head Neck Surg. 1994;110:222-7.

31. Shahan TK, Logemann JA, Rademaker AW, Paulosky BR, Kahrilas PJ. Chin-down posture effect on aspiration in dysphagic patients. Arch Phys Med Rehabil. 1993;74:736-9.

Ludmiła Halczy-Kowalik MD

Mieczysław Sulikowski MD

Rościsław Wysocki MD

Violetta Posio MD

Robert Kowalczyk MD

Anna Rzewuska MD 